\title{
Ginsenoside Rg1 improves cognitive capability and affects the microbiota of large intestine of tree shrew model for Alzheimer's disease
}

\author{
YUQIAN GUO $^{1 *}$, LIMEI WANG $^{1 *}$, JIANGLI LU $^{1}$, JIANLIN JIAO $^{2}$, YI YANG $^{1}$, \\ HONGBIN ZHAO $^{3}$, ZHANG LIANG $^{4}$ and HONG ZHENG ${ }^{1}$ \\ ${ }^{1}$ Department of Laboratory Animal Science and ${ }^{2}$ Technology Transfer Center, Kunming Medical University, \\ Kunming, Yunnan 650500; ${ }^{3}$ Department of Emergency Medicine, First Affiliated Hospital of Kunming Medical University, \\ Kunming, Yunnan 650031; ${ }^{4}$ Research Management Office for Science and Technology, \\ Kunming Medical University, Kunming, Yunnan 650500, P.R. China
}

Received May 14, 2020; Accepted December 10, 2020

DOI: $10.3892 / \mathrm{mmr} .2021 .11931$

\begin{abstract}
Ginsenoside $\operatorname{Rg} 1(\operatorname{Rg} 1)$ is traditional Chinese medicine with neuroprotective activity. Previous studies have demonstrated that $\mathrm{Rg} 1$ improves Alzheimer's disease (AD) and alters gut microbiology, but its mechanism remains to be elucidated, and thus far, its use in the treatment of AD has not been satisfactory. The present study investigated the improvement effects of $\operatorname{Rg} 1$ and its association with the microbiota of the large intestine. Following treatment with $\mathrm{Rg} 1$ in $\mathrm{AD}$ tree shrews, the treatment group demonstrated significantly shorter escape latency and crossed a platform more frequently in a water maze test. Western blotting demonstrated that Rg1 inhibited the expression of $\beta$-secretase 1 , while increasing microtubule-associated protein 2 and Fox-3 in the hippocampus. Immunohistochemical analysis revealed that $\mathrm{Rg} 1$ decreased the expression of amyloid $\beta$, tau phosphorylated at serine 404 and pro-apoptotic factor Bax, while increasing the expression of Bcl-2 in the hippocampus and cortex. High throughput sequencing of $16 \mathrm{~S}$ rRNA demonstrated that Rg1 altered the microbiota abundance of the large intestine. In
\end{abstract}

Correspondence to: Mrs. Zhang Liang, Research Management Office for Science and Technology, Kunming Medical University, 1168 West Chunrong Road, Chenggong, Kunming, Yunnan 650500, P.R. China

E-mail: ynkml-z@163.com

Professor Hong Zheng, Department of Laboratory Animal Science, Kunming Medical University, 1168 West Chunrong Road, Chenggong, Kunming, Yunnan 650500, P.R. China

E-mail: zhengandhong@126.com

*Contributed equally

Key words: Alzheimer's disease, tree shrew, microbiota of large intestine, ginsenoside $\mathrm{Rg} 1$, neuroprotection conclusion, $\mathrm{Rg} 1$ affected the expression of apoptosis proteins, possessed a neuroprotective effect and may have a close association with the microbiota of large intestine by significantly reducing the abundance of Bacteroidetes and increasing the energy requirement of tree shrews.

\section{Introduction}

Alzheimer's disease (AD), which is pathologically manifested as amyloid $\beta(\mathrm{A} \beta)$ deposition and the formation of neurofibrillary tangles (NFTs) (1), accompanied by the decrease of memory, judgment, attention and cognitive ability, is a neurodegenerative disorder (2). It has a serious effect on the quality of life of patients. Thus far, there are no effective means to prevent or treat $\mathrm{AD}(3)$.

Ginsenoside Rg1 (Rg1) exhibits anti-inflammatory, antioxidant, antitumor and anti-apoptosis effects $(4,5)$. The effects of $\operatorname{Rg} 1$ on memory enhancement and neuroprotection have attracted wide attention (6,7). Rg1, which inhibits $A \beta$ plaques and has anti-inflammatory and anti-oxidation effects, can improve the learning and memory ability of AD mice (6). In vitro experiments, by accelerating the activation and nuclear translocation and transcription of $N F-\kappa B, R g 1$ increased the binding of $\mathrm{NF}-\kappa \mathrm{B}$ with the corresponding DNA sites at the promoter region of the $\beta$ secretase gene, inhibiting the transcription and translation of $\beta$ secretase and thus reducing the production of $\mathrm{A} \beta$ (7).

The microbiota of the large intestine is a complex microecosystem in the host and its stability is closely related to the health of the body (8). Intestinal microbial composition and the communication between microbes and the brain may change with age and the microbial composition of those $>65$ years old is significantly different from those $<9$ years old (9-11). Intestinal symptoms have been observed in patients with neurodegenerative diseases and mental disorders, including Parkinson's disease, autism, schizophrenia, Alzheimer's disease and depression (12-15).

Previous studies have confirmed that AD is closely associated with the composition of intestinal microflora. 
For example, bacterial metabolites, such as short-chain fatty acids, may mediate the maturation of microglia (16) and further result in the clearance of $A \beta$ and the protection of neurons (17). When rats aged 22.5-25 months were exposed to $E$. coli able to produce curly amyloid fibrils, the deposition of $\mathrm{A} \beta$ in the gut and brain neurons increased and the proliferation of glial cells and astrocytes was enhanced (18). Antibiotics, which interfere with the intestinal microbial diversity of $A D$ mice, significantly reduce the deposition of $A \beta$ and glial cells hyperplasia (19). 16S rRNA sequencing of fecal samples of amyloid precursor protein (APP)/presenilin 1 transgenic mice and wild-type mice demonstrates a significant difference in the composition of intestinal flora between the two groups of mice (20). Zhang et al (8) demonstrated that, compared with the wild-type mice, AD mice had more diversified intestinal bacteria, a lower ratio of Firmicutes to Bacteroides and more Proteus and Verrucous.

The effect of traditional Chinese medicine Rg1 on intestinal bacteria has become a focus of medical research. Poria cocos water-insoluble polysaccharide can adjust intestinal flora in mice, increasing Helicobacter and Clostridium and further improving hyperglycemia and hyperlipidemia (21). Following long-term use of ginseng extract, the number of probiotics such as Bifidobacterium, Lactobacillus and Clostridium was significantly increased in the intestinal flora of rats, suggesting that it can promote the growth of probiotics (22). Tiansi solution, a traditional Chinese herbal medicine, which increases the relative abundance of Ruminococcaceae, Lactococcus and Lactobacillus, significantly improves the exploratory behavior of depressed rats (23).

The Chinese tree shrew (Tupaia belangeri chinensis) is considered a 'low-level primate' based on its relation to primates (24). There is a genetic basis for using tree shrew as a viable neurological disease model (25). The tree shrew model of AD has been successfully constructed in our study (26). A recent study has suggested that the neocortex development of the tree shrew is closer to primates than to rodents (27). This makes the tree shrew a good experimental animal in neurological diseases.

The present study investigated the influence of Rg1 on the expression of $A \beta$-related proteins, phosphorylated (p-)tau and apoptosis-related protein including Bax, Bcl-2, microtubuleassociated protein 2 (MAP2) and Fox-3 (NeuN), in AD tree shrews and further analyzed the microbiota of their large intestine.

\section{Materials and methods}

Ethics statement. The present study was performed in strict accordance with the Health Guide for the Care and Use of Laboratory Animals (28). The protocol was approved by the Committee on the Ethics of Animal Experiments of Kunming Medical University (approval number KMMU2018004).

Animals. Tree shrews were purchased from the Department of Experimental Animals of Kunming Medical University. As female tree shrews menstruate, only males were used to avoid the influence of menstruation. A total of 15 adult male tree shrews were used (10-12 months; weight range, 130-150 g).
The tree shrews were housed in independent ventilation cages at an ambient temperature of $24 \pm 2^{\circ} \mathrm{C}$ and a humidity of 40-60\% under a 12-h light/dark cycle with free access to food and water at Kunming Medical University. All the animals were acclimated to their new habitat for a week prior to the experiments.

The tree shrews were divided into the model group $(\mathrm{D}+\mathrm{A})$, the control group (CT) and the treatment group $(\mathrm{D}+\mathrm{A}+\mathrm{Rg} 1)$, with five tree shrews in each group. The $\mathrm{D}+\mathrm{A}$ group received one-time lateral ventricle injection $(0.8 \mathrm{~mm}$ posterior to the bregma, $1.5 \mathrm{~mm}$ lateral to the sagittal suture $3.6 \mathrm{~mm}$ beneath the surface of the brain; the endocranium was exposed by drilling the skull, and a micro syringe inserted vertically for injection. A $\beta 25-35(10 \mu \mathrm{g} / 5 \mu \mathrm{l}$; cat. no. A9810; Sigma-Aldrich; Merck KGaA) and D-galactose (60 mg/kg/day; cat. no. A-G5388; Sigma-Aldrich; Merck $\mathrm{KGaA})$ were injected subcutaneously, using a syringe for 6 weeks (29). Ginsenoside Rg1 (Kunming Pharmaceutical Group Co., Ltd.) was administered to the treatment group (30 $\mathrm{mg} / \mathrm{kg} /$ day) following the basis of the administration modeling method in the $\mathrm{D}+\mathrm{A}$ group. The control group was given an equal amounts of saline and the injection site was the same as for A $\beta 25-35$ in the model group. Following the Morris water maze tests, all shrews were sacrificed by exsanguination and the cerebral hemisphere was obtained; one hemisphere was perfused with $4 \%$ paraformaldehyde $\left(4^{\circ} \mathrm{C}\right)$ overnight and the hippocampus and cortex taken for immunohistochemistry. The other hemisphere was frozen at $-80^{\circ} \mathrm{C}$ for western blotting. The content of the large intestine was obtained and snap-frozen in liquid nitrogen and stored at $-80^{\circ} \mathrm{C}$ for $16 \mathrm{SrRNA}$ analysis.

Morris water maze. A black circular pool of $160 \mathrm{~cm}$ diameter with a platform in its center was used. Water was poured into the pool to a depth of $\sim 40 \mathrm{~cm}$, then milk powder was scattered to make the water opaque. The water temperature was maintained at $22 \pm 1^{\circ} \mathrm{C}$ and the platform height at $\sim 11 \mathrm{~cm}$. A tracking camera located directly above the water was used to record the position, swimming time and paths of the tree shrews. The first day was adaptation training, with the water maze arranged with a visible platform. Tree shrews were held to face the wall of the pool and dropped down from a height of $10 \mathrm{~cm}$ above the water surface. On the first day's adaptation training, if the platform was not found after $30 \mathrm{sec}$, the tree shrew would be guided to the platform and then be kept there for $10 \mathrm{sec}$. The tree shrew was taken out and wiped dry, and the procedure repeated for two rounds over 2-6 days for the positioning cruise experiment. In order to hide the platform, it was placed $1 \mathrm{~cm}$ below the water surface. The positioning cruise experiment was performed for 2-6 days. The same operation as the first day was performed to record how long it took the tree shrew to find the platform. In the spatial search experiment (7th day), the platform which was used in the experiment in the previous 7 days was removed, and the number of times that the tree shrews crossed the platform were recorded. Software (Morris water maze: cat. no. XR-XM101; Shanghai Xin-Xin soft Information Technology Co., Ltd.) recorded the escape latency and the frequency of crossing the platform of tree shrews. GraphPad Prism 8.0.2 (GraphPad Software, Inc.) was used for analysis. 
Western blot analysis. Total proteins of hippocampal tissues were extracted by using a protein extraction kit (cat. no. R0020; Beijing Solarbio Science \& Technology Co., Ltd.). The lysate was added proportionally as $200 \mu \mathrm{l}$ lysate per $20 \mathrm{mg}$ of tissue and seahorse tissue was homogenized with glass homogenizer until fully lysed. The lysate was centrifuged for 3-5 min at 5,000 x g and $-4^{\circ} \mathrm{C}$ and the supernatant removed. Hippocampal protein concentration was determined by the BCA protein concentration assay kit (cat. no. PC0020; Beijing Solarbio Science \& Technology Co., Ltd.). The supernatant of the protein sample was heated at $95^{\circ} \mathrm{C}$ for $10 \mathrm{~min}$. Sodium dodecyl sulfate-polyacrylamide gel $(10 \%)$ was prepared by a SDS-page gel preparation kit with a sample amount of $40 \mu \mathrm{g}$, and then electrophoresed for $30 \mathrm{~min}$ at $80 \mathrm{~V}$ and $1.5 \mathrm{~h}$ at $120 \mathrm{~V}$. Protein bands transferred onto polyvinylidene fluoride membranes and blocked with 5\% skimmed milk at room temperature for $1 \mathrm{~h}$. The membranes were incubated overnight at $4^{\circ} \mathrm{C}$ with antibodies against MAP2 (1:1,000; Cell Signaling Technology, Inc.; cat. no. 8707), NeuN (1:1,000; Cell Signaling Technology, Inc.; cat. no. 24307), $\beta$-secretase 1 (BACE1; 1:500; Abcam; cat. no. ab183612) and $\beta$-actin $(1: 1,000$; Elabscience, Inc.; cat. no. 20031). The secondary antibody [peroxidase-conjugated goat anti-rabbit IgG (H+L); 1:5000; OriGene Technologies, Inc.; cat. no. ZB-2301] was incubated for $1 \mathrm{~h}$ and then developed with ECL reagent (ECL Plus Ultra Sensitive kit; Phygene Life Sciences; cat. no. PH0353). Chemiluminescence signals were imaged by a Gel Imaging System (Bio-Rad Laboratories, Inc.) and protein band signals are quantified by ImageJ software (v1.8.0; National Institutes of Health).

Immunohistochemical analysis of hippocampus and cortex. The cerebral hemisphere was fixed in $4 \%$ paraformaldehyde for $48 \mathrm{~h}$ at room temperature, rinsed under tap water for $1.5 \mathrm{~h}$, changed to $70 \%$ alcohol and soaked for $2 \mathrm{~h}$, changed to $80 \%$ alcohol and soaked for $2 \mathrm{~h}$, changed to $90 \%$ alcohol and soaked for $1.5 \mathrm{~h}$, changed to $95 \%$ alcohol and soaked for $1.5 \mathrm{~h}$, changed to $100 \%$ alcohol and soaked for $45 \mathrm{~min}$, changed to another $100 \%$ alcohol and soaked for $45 \mathrm{~min}$, changed to xylene and soaked for $15 \mathrm{~min}$, changed to another $100 \%$ xylene and soaked for 15 min, changed to paraffin and soaked for $4 \mathrm{~h}$ before being cut into coronal sections at $5 \mu \mathrm{m}$ thickness and warmed for $30 \mathrm{~min}$. The sections were dewaxed (xylene 1 for $10 \mathrm{~min}$, xylene 2 for $10 \mathrm{~min}, 100 \%$ ethanol 1 for $3 \mathrm{~min}, 100 \%$ ethanol 2 for $3 \mathrm{~min}, 95 \%$ ethanol $3 \mathrm{~min}$, $90 \%$ ethanol $3 \mathrm{~min}, 80 \%$ ethanol $3 \mathrm{~min}, 70 \%$ ethanol $3 \mathrm{~min}$ and distilled water $3 \mathrm{~min}$ ). Following heating with citric acid antigen repair solution in a pressure cooker $\left(100^{\circ} \mathrm{C}\right)$ for 2 min the sections were washed with PBS for three times, 3 min per time. The sections were blocked with $3 \%$ hydrogen peroxide for $10 \mathrm{~min}$ at room temperature and washed with PBS for three times, 3 min per time. Primary antibodies A $\beta 1-42$ (1:200; cat. no. ab2539; Abcam), APP (1:4,000; SigmaAldrich; Merck KGaA; cat. no. SAB4300464), p-tau (1:1,000; cat. no. ab92676; Abcam), Bax (1:1,000; cat. no. 5023; Cell Signaling Technology, Inc.), Bcl-2 (1:1,000; cat. no. 4223; Cell Signaling Technology, Inc.) were added and the sections placed into a $4^{\circ} \mathrm{C}$ refrigerator overnight. Taken out of the $4^{\circ} \mathrm{C}$ refrigerator, the sections were rinsed with PBS for three times, 3 min per time and secondary antibody (goat anti-rabbit IgG;
1:5,000; cat. no. AP132; Sigma-Aldrich; Merck KGaA) added and incubated for $15 \mathrm{~min}$ at room temperature. PBS was used to rinse for 3 times at 3 min per time. DAB was used for staining for $4 \mathrm{~min}$ and hematoxylin counterstaining for $2 \mathrm{~min}$ at room temperature. After washing in water for several seconds the sections were differentiated in hydrochloric acid and alcohol. The sections were dehydrated (a gradient of $70 \%$ ethanol, $80 \%$ ethanol, $90 \%$ ethanol, $95 \%$ ethanol, $100 \%$ ethanol 1 , and $100 \%$ ethanol for $1 \mathrm{~min}$, and xylene 1 and xylene 2 for $10 \mathrm{~min}$ ), sealed and allowed to dry. A total of three sections were taken randomly from each animal, five animals in each group. The staining intensity over the entire surface area of the slide was calculated using ImageJ software (v.1.51j8; National Institutes of Health).

Analysis microbiota of large intestine. The contents of the large intestine were extracted by using a DNA extraction kit (E.Z.N.A. ${ }^{\circledR}$ Stool DNA kit; cat. no. D4015-01; Omega Bio-Tek, Inc.) and the bacterial genome was extracted according to the manufacturer's protocols. The bacterial genomic DNA of large intestine contents among groups D+A, CT and D+A+Rg1 were detected by $1 \%$ agarose gel electrophoresis. The sequencing primers 341F (5'-CCTAYGGGRBGCASCAG-3') and 806R (5'-GGACTACNNGGGTATCTAAT-3') were used to amplify V3-V4 region of bacterial 16S rDNA gene sequence using the sequencing kit HiSeq Rapid SBS Kit v2 that performed in accordance with the manufacturer's protocols. (cat. no. FC-402-4022; Illumina, Inc.).

PCR products were quantified by QuantiFluor ${ }^{\mathrm{TM}}-\mathrm{ST}$ blue fluorescence quantitative system (Promega Corporation). An Illumina PE250 library was built (Illumina, Inc.): ' $\mathrm{Y}$ ' sticky joints were connected, self-connecting segments were removed by magnetic bead screening, the template libraries were enriched by PCR, and the single-stranded DNA fragments were produced by sodium hydroxide. The database, obtained from the Illumina PE250 platform (Illumina, Inc.), was uploaded to NCBI Sequence Read Archive (SRP: 227299).

In order to get corresponding species classification information from each operational taxonomic unit (OTU), the present study used the RDP Classifier (v 2.2; http://sourceforge.net/ projects/rdp-classifier/) Bayesian algorithm on the $97 \%$ similarity level OTU to characterize taxonomy sequence analysis, at each classification level (domain, kingdom, phylum, class, order, family, genus and species) for the community composition of each sample.

The Chao, Shannon and diversity indices were analyzed based on Mothur (v.1.30.1 http://www.mothur.org). Linear discriminant analysis effect size (LEfSe) uses linear discriminant analysis (LDA) to estimate the effect of abundance of each component (species) on different effects. LDA is performed according to different grouping conditions according to taxonomic composition to identify the communities or species that have a significant influence on sample division.

Shannon: One of the microbial diversity indexes used to estimate microbial diversity in samples. It is often used with the Simpson diversity index to reflect the Alpha diversity index. Higher Shannon values indicate higher community diversity.

$$
H_{\text {shannon }}=-\sum_{i=1}^{S_{\text {obs }}} \frac{n_{i}}{N} \ln \frac{n_{i}}{N}
$$


$S_{o b s}=$ the actual number of OTUs measured; $n_{i}=$ the sequence number contained in the ith OTU; $N=$ the number of sequences.

Chao: This is an index to estimate the number of OTU in a sample by using the chaol algorithm. Chaol is often used in ecology to estimate the total number of species and was first proposed by Chao (30). The calculation formula used in this analysis is as follows:

$$
S_{\text {chao } 1}=S_{o b s}+\frac{n_{1}\left(n_{1}-1\right)}{2\left(n_{2}+1\right)}
$$

$S_{\text {chaol }}=$ Estimated OTU number; $S_{\text {obs }}=$ actual observed OTU number; $n_{l}=$ number of OTUs containing only one sequence (e.g. 'Singletons'); $\mathrm{n}_{2}=$ number of OTUs containing only two sequences (e.g. 'Doubletons').

Statistical analysis. All data are expressed as mean \pm standard deviation or arithmetical mean. Differences among groups were analyzed by one-way analysis of variance (ANOVA) followed by Tukey's post hoc test. Statistical analyses were performed using SPSS v21.0 software (IBM Corp.) except for Fig. 1A, which was analyzed by two-way ANOVA followed by Tukey's post hoc test. GraphPad Prism 8.0.2 (GraphPad Software, Inc.) software was used for the statistical graphs. Metastats (http://metastats.cbcb.umd.edu/) difference analysis based on the data of community abundance detected the differences of the two groups of microbial communities in abundance, and performed multiple hypothesis tests analysis, assess the significance of the observed differences. $\mathrm{P}<0.05$ was considered to indicate a statistically significant difference.

\section{Results}

Rgl improves learning and memory capacity in AD tree shrews. A Morris water maze was used to detect the escape latency period (2-6 days) and the times of crossing the platform ( 7 th day) of tree shrews in the 3 groups. The results demonstrated that the evasive incubation period of group $\mathrm{D}+\mathrm{A}+\mathrm{Rg} 1$ was significantly lower compared with group $\mathrm{D}+\mathrm{A}$ $(\mathrm{P}<0.01)$ on days 4-6 (Fig. 1A) and no significantly difference was observed among the individual groups on days 4-6. The number of times of crossing the platform of group $\mathrm{D}+\mathrm{A}+\mathrm{Rg} 1$ was significantly greater compared with group $\mathrm{D}+\mathrm{A}(\mathrm{P}<0.01$; Fig. 1B).

Rg1 reduces production of A $\beta 1-42$ and phosphorylation of tau. Immunohistochemistry demonstrated no significant difference in the expression of APP protein in the hippocampus and cortex (Fig. 2A and C). Western blot analysis demonstrated that the expression of BACE1 protein was significantly increased in the hippocampus of the $\mathrm{D}+\mathrm{A}$ group compared with the CT group $(\mathrm{P}<0.01$; Fig. $2 \mathrm{~B}$ and $\mathrm{D})$. The expression of BACE1 protein was significantly lower in the hippocampus of group $\mathrm{D}+\mathrm{A}+\mathrm{Rg} 1$ compared with group $\mathrm{D}+\mathrm{A}(\mathrm{P}<0.01$; Fig. $2 \mathrm{~B}$ and $\mathrm{D})$. The expression of $A \beta 1-42$ in the hippocampus and cortex of group $\mathrm{D}+\mathrm{A}+\mathrm{Rg} 1$ was significantly lower compared with group $\mathrm{D}+\mathrm{A}(\mathrm{P}<0.01$; Fig. $2 \mathrm{E}$ and $\mathrm{G})$. Immunohistochemistry demonstrated that the expression of tau phosphorylated at serine 404 (pS404-tau) in the hippocampus and cortex of group D+A was significantly higher compared with the CT group $(\mathrm{P}<0.01)$.
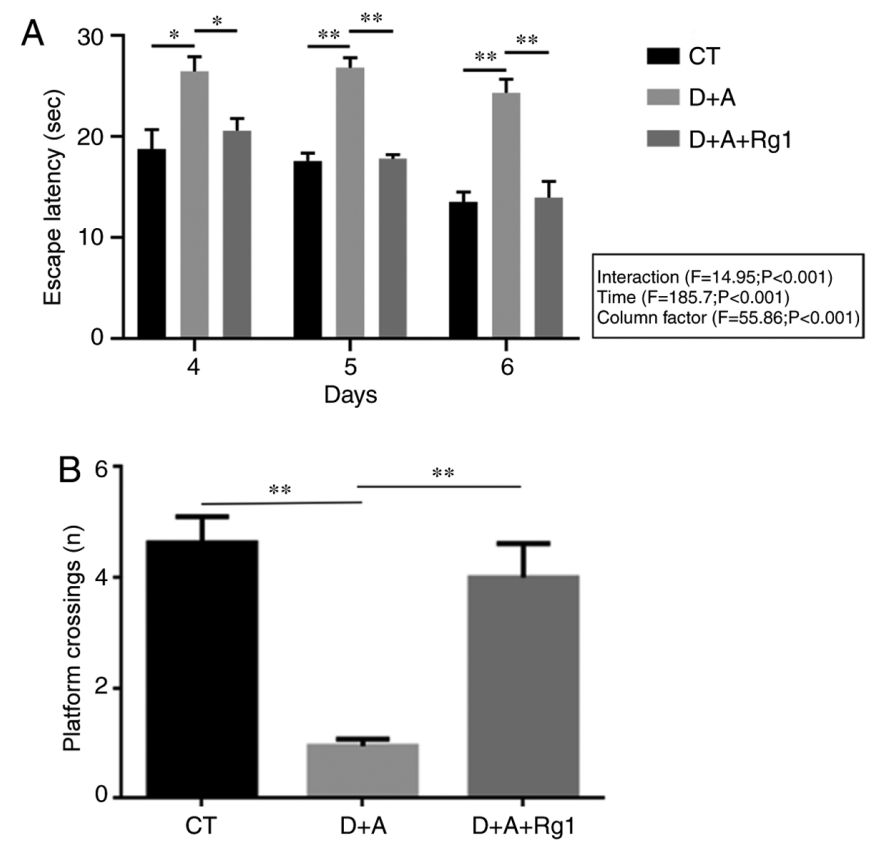

Figure 1. Statistical results of the Morris water maze. (A) The escape latency of AD tree shrews on days 4-6 in the three groups. (B) The number of times tree shrews crossed the platform on day 7 in the three groups. ${ }^{*} \mathrm{P}<0.05$, ${ }^{* *} \mathrm{P}<0.01$. AD, Alzheimer's disease; CT, control group; $\mathrm{D}+\mathrm{A}$, model group; $\mathrm{D}+\mathrm{A}+\mathrm{Rg} 1$, treatment group; Rg1, ginsenoside Rg1.

Rg1 significantly inhibited the phosphorylation of pS404-tau in the hippocampus and cortex $(\mathrm{P}<0.01$; Fig. $2 \mathrm{~F}$ and $\mathrm{H})$.

Rg1 increases the expression of anti-apoptotic factor Bcl-2 and decreases the expression of pro-apoptotic factor Bax. Immunohistochemistry of the hippocampus and cortex demonstrated that the expression of $\mathrm{Bcl}-2$ protein in the D+A group was significantly decreased compared with the CT group $(\mathrm{P}<0.01)$. Compared with the $\mathrm{D}+\mathrm{A}$ group $(\mathrm{P}<0.01)$, $\mathrm{Rg} 1$ significantly increased the expression of $\mathrm{Bcl}-2$ protein in the $\mathrm{D}+\mathrm{A}+\mathrm{Rg} 1$ group $(\mathrm{P}<0.01$; Fig. $3 \mathrm{~A}$ and $\mathrm{C})$, while it significantly decreased the expression of Bax protein in the $\mathrm{D}+\mathrm{A}+\mathrm{Rg} 1$ group $(\mathrm{P}<0.01$; Fig. $3 \mathrm{~B}$ and $\mathrm{D})$.

The neuron effects of $\mathrm{Rg} 1$ on the hippocampus and cortex. Western blot analysis demonstrated that the expression of MAP2 and NeuN in tree shrews were similar. The expression of MAP2 $(\mathrm{P}<0.01)$ and NeuN $(\mathrm{P}<0.01)$ in the $\mathrm{D}+\mathrm{A}$ group were significantly decreased compared with the CT group, Rg1 significantly increased expression of MAP2 ( $<<0.01$; Fig. 4A and C) and NeuN ( $\mathrm{P}<0.01$; Fig. 4B and D). Immunohistochemistry demonstrated that NeuN expression in the cortex and hippocampus demonstrated that the positive cell rate and area in the $\mathrm{D}+\mathrm{A}+\mathrm{Rg} 1$ group were significantly increased compared with the $\mathrm{D}+\mathrm{A}$ group $(\mathrm{P}<0.01 ;$ Fig. $4 \mathrm{E}$ and $\mathrm{F})$.

Rgl changes the microbiota of large intestine. A total of 398,382 sequences were acquired in the microbiota of large intestine contents samples. OTUs were mapped at $97 \%$ similarity. The coverage index of nine samples was $>0.999$, which meant that the sequencing results contained $>99.9 \%$ of the bacterial diversity in the large intestine (Fig. 5A). A Venn diagram demonstrated that, among all 353 OTUs, all 

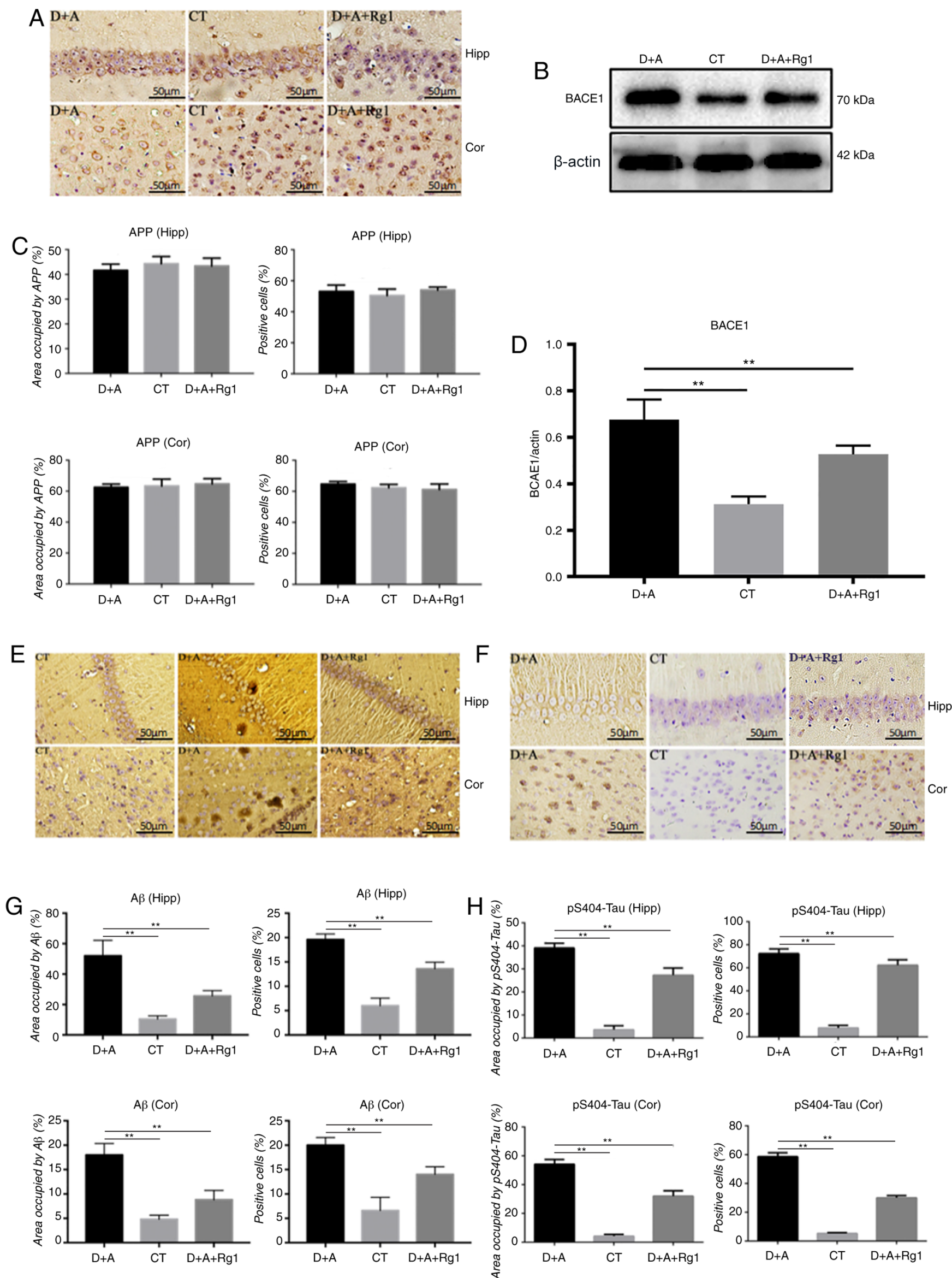

Figure 2. Effect of ginsenoside Rg1 on A $\beta 1-42$ associated protein and pS404-tau expression in AD tree shrews. Immunohistochemical analysis of (A and C) APP, (E and G) A $1-42$ and (F and H) pS404-tau in the hippocampus and cortex. Western blot analysis of (B and D) BACE1 in the hippocampus and cortex. ${ }^{* *} \mathrm{P}<0.01$. A $\beta$, amyloid $\beta ; \mathrm{pS} 404$-tau, tau phosphorylated at serine 404; AD, Alzheimer's disease; APP, amyloid precursor protein; CT, control group; $\mathrm{D}+\mathrm{A}$, model group; D+A+Rg1, treatment group; Rg1, ginsenoside Rg1; Hipp, hippocampus; cor, cortex; BACE1, $\beta$-secretase 1. 
A
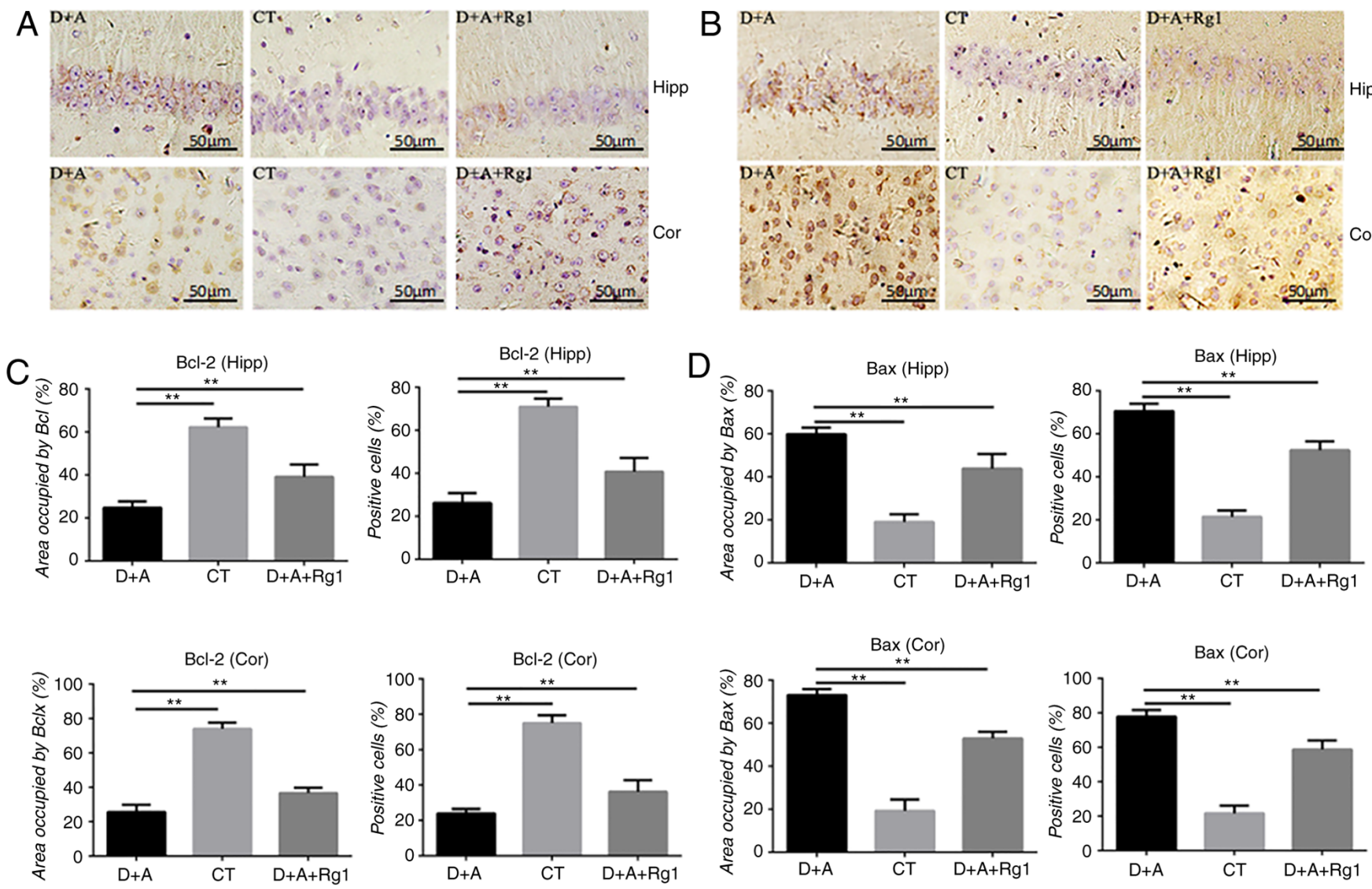

Figure 3. Effect of ginsenoside Rg1 on Bcl-2 and Bax protein expression in AD tree shrews. Immunohistochemistry analysis of (A and C) Bcl-2 and (B and D) Bax in the cortex and hippocampus. ${ }^{* *} \mathrm{P}<0.01$. AD, Alzheimer's disease; CT, control group; D+A, model group; D+A+Rg1, treatment group; Rg1, ginsenoside Rg1; Hipp, hippocampus; cor, cortex.

Table I. Composition of intestinal flora in three groups of tree shrews at the level of phylum and class.

\begin{tabular}{lccc}
\hline Groups & Bacteroidetes & Betaproteobacteria & Negativicutes \\
\hline D+A & $0.2867 \pm 0.1381^{\mathrm{a}}$ & $0.2866 \pm 0.1380^{\mathrm{a}}$ & $0.0807 \pm 0.0502^{\mathrm{b}}$ \\
$\mathrm{CT}$ & $0.0255 \pm 0.0306$ & $0.0246 \pm 0.0307$ & $0.0033 \pm 0.0031$ \\
$\mathrm{D}+\mathrm{A}+\mathrm{Rg} 1$ & $0.0486 \pm 0.0428$ & $0.0471 \pm 0.0449$ & $0.0045 \pm 0.0027$ \\
\hline
\end{tabular}

The D+A group was significantly different from the other two groups. There were significant differences between D+A group and the other two groups. ${ }^{\mathrm{P}}<0.01$ and ${ }^{\mathrm{b}} \mathrm{P}<0.05$ vs. $\mathrm{CT}$ and $\mathrm{D}+\mathrm{A}+\mathrm{Rg} 1$ groups. $\mathrm{D}+\mathrm{A}$, model group; CT, control group; $\mathrm{D}+\mathrm{A}+\mathrm{Rg} 1$, treatment group; $\mathrm{Rg} 1$, ginsenoside $\mathrm{Rg} 1$.

samples shared 138 OTUs; the CT group was 32 OTUs, the $\mathrm{D}+\mathrm{A}$ group was 46 OTUs and the $\mathrm{D}+\mathrm{A}+\mathrm{Rg} 1$ was 32 OTUs (Fig. 5B). These results suggested that the bacteria species in $\mathrm{D}+\mathrm{A}+\mathrm{Rg} 1$ group were similar to the $\mathrm{CT}$ group. The larger the Chao value, the larger total number of species; the larger Shannon value, the higher community diversity (Fig. 5C and D). No significant difference was observed between the Chao and Shannon indexes among the three groups $(\mathrm{P}>0.05)$. The $\mathrm{D}+\mathrm{A}$ group exhibited higher Chao and Shannon indexes compared with the CT group, while the results from the $\mathrm{D}+\mathrm{A}+\mathrm{Rg} 1$ group were closer to the $\mathrm{CT}$ group. LEfSe analysis revealed nine genera that were differentially represented in the $\mathrm{CT}$ group compared with other groups; and there were 8 genera in the $\mathrm{D}+\mathrm{A}$ group and six genera in the $\mathrm{D}+\mathrm{A}+\mathrm{Rg} 1$ group $(\mathrm{P}<0.05$; Fig. 5E). The abundance of Lactobacillus salivarius in the D+A group was the lowest among the groups and highest in the CT group. The abundance of Lactobacillus salivarius in the $\mathrm{D}+\mathrm{A}+\mathrm{Rg} 1$ group was higher than $\mathrm{D}+\mathrm{A}$ group, which indicated that $\mathrm{Rg} 1$ could increase the abundance of Lactobacillus salivarius in the intestinal flora of AD tree shrews (Fig. 5F).

Meta Stat difference analysis at the phylum level demonstrated that the abundance of Bacteroidetes in the $\mathrm{D}+\mathrm{A}$ group was significantly higher compared with the $\mathrm{D}+\mathrm{A}+\mathrm{Rg} 1$ group $(\mathrm{P}<0.01)$; no significant difference was observed between the $\mathrm{D}+\mathrm{A}+\mathrm{Rg} 1$ and CT groups(Table I). The Firmicutes/Bacteroides ratio of the D+A and CT group was 1.3:24.7 and the Firmicutes/ Bacteroides ratio in the $\mathrm{D}+\mathrm{A}$ and $\mathrm{D}+\mathrm{A}+\mathrm{Rg} 1$ group was 1.3:9. 

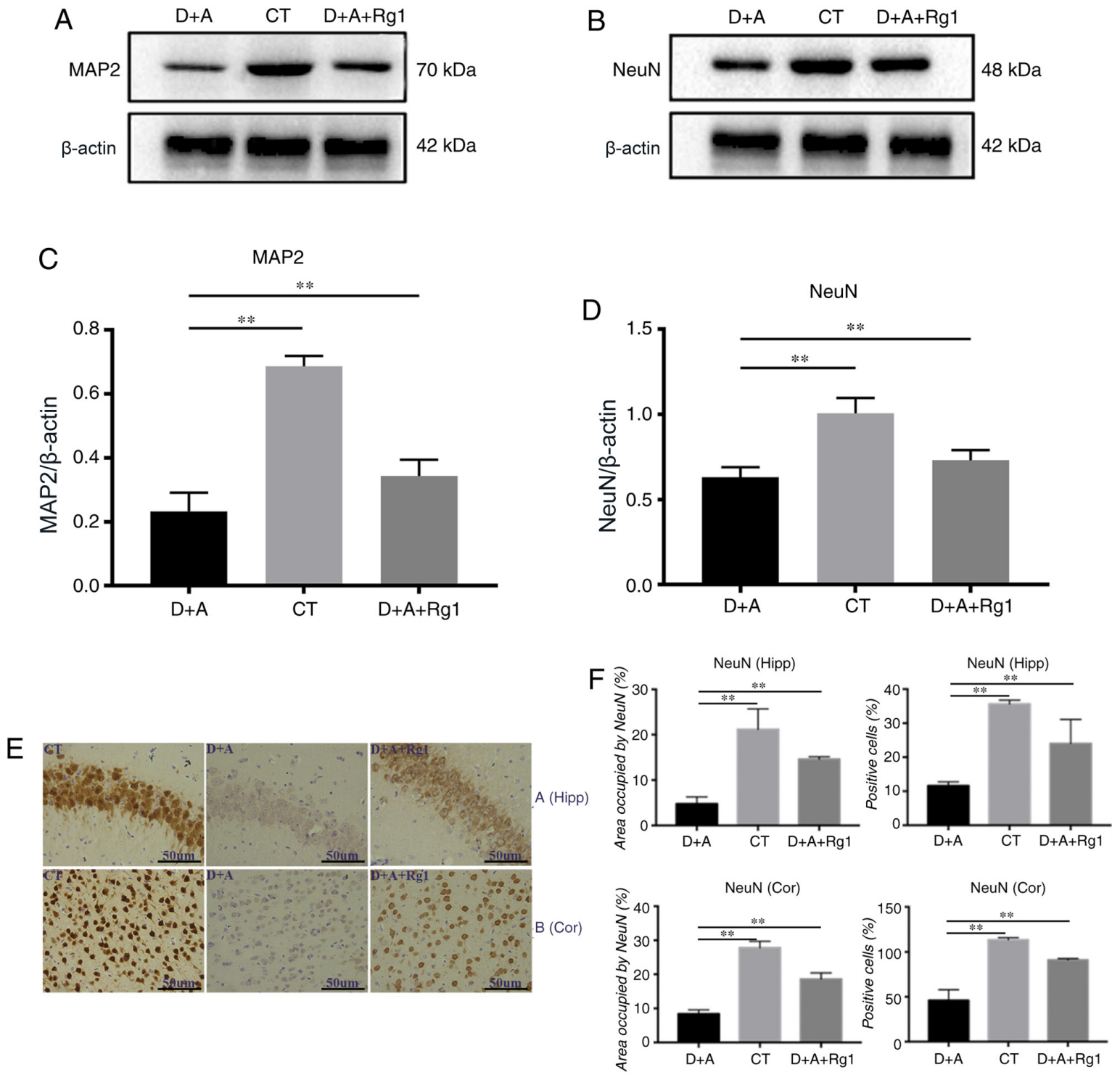

Figure 4. Effect of ginsenoside Rg1 on MAP2 and NeuN expression in AD tree shrews. Western blot analysis of (A and C) MAP2 and (B and D) NeuN in the hippocampus. (E and F) Immunohistochemistry analysis of NeuN in cortex and hippocampus. ${ }^{* *} \mathrm{P}<0.01$. AD, Alzheimer's disease; MAP2, microtubuleassociated protein 2; NeuN, Fox-3; D+A, model group; CT, control group; D+A+Rg1, treatment group; Rg1, ginsenoside Rg1.

The ratio of Firmicutes/Bacteroidetes in tree shrews in the $\mathrm{D}+\mathrm{A}$ group was only 0.0526 times higher than the CT group. Following Rg1 treatment, the Firmicutes/Bacteroides ratio in the intestinal flora of tree shrews increased to 6.923 times that of the D+A group. At the class level, the abundance of Bacteroidia in group D+A was significantly higher compared with the CT group $(\mathrm{P}<0.01)$ and the $\mathrm{D}+\mathrm{A}+\mathrm{Rg} 1$ group $(\mathrm{P}<0.01)$, while no significant difference was observed between groups $\mathrm{D}+\mathrm{A}+\mathrm{Rg} 1$ and CT (Table I). The abundance of Negativicutes in the $\mathrm{D}+\mathrm{A}$ group was higher in the $\mathrm{CT}(\mathrm{P}<0.05)$ and the $\mathrm{D}+\mathrm{A}+\mathrm{Rg} 1$ groups $(\mathrm{P}<0.05)$, but no significant difference was observed between the D+A+Rg1 and CT groups (Table I).

\section{Discussion}

Rgl may improve the cognitive function of $A D$ tree shrews by interfering with A $\beta 1-42$ formation and reducing tau phosphorylation. In the present study, the escape latency decreased and the number of times the platform was crossed increased following treatment with $\mathrm{Rg} 1$. Therefore, $\mathrm{Rg} 1$ enhanced learning and memory ability in AD tree shrews. The spatial memory ability of mice following the Rg1 treatment group was improved and the aggregation level of $\mathrm{A} \beta$ was significantly reduced (31).

The pathological changes of $\mathrm{AD}$ are extracellular senile plaques formed by the deposition of $\mathrm{A} \beta$ and intracellular neurofibrillary tangles formed by excessive phosphorylation of tau protein (19). APP is produced by the shear of BACE1 and $\gamma$-secretase (32). The majority of patients with AD have elevated levels of BACE1, which leads to the production of $\beta$-amyloid. BACE1 may be a potential biomarker and therapeutic target for AD $(33,34)$. Du et al (31) found that mice treated with Rg1 possess significantly reduced levels of $A \beta$ aggregation and tau phosphorylation. In general, Rg1 can prevent AD by reducing the production of $\mathrm{A} \beta$ and the phosphorylation of tau (35).

Rg1 exhibits a neuroprotective effect on AD tree shrews by inhibiting the expression of pro-apoptotic proteins. MAP2 

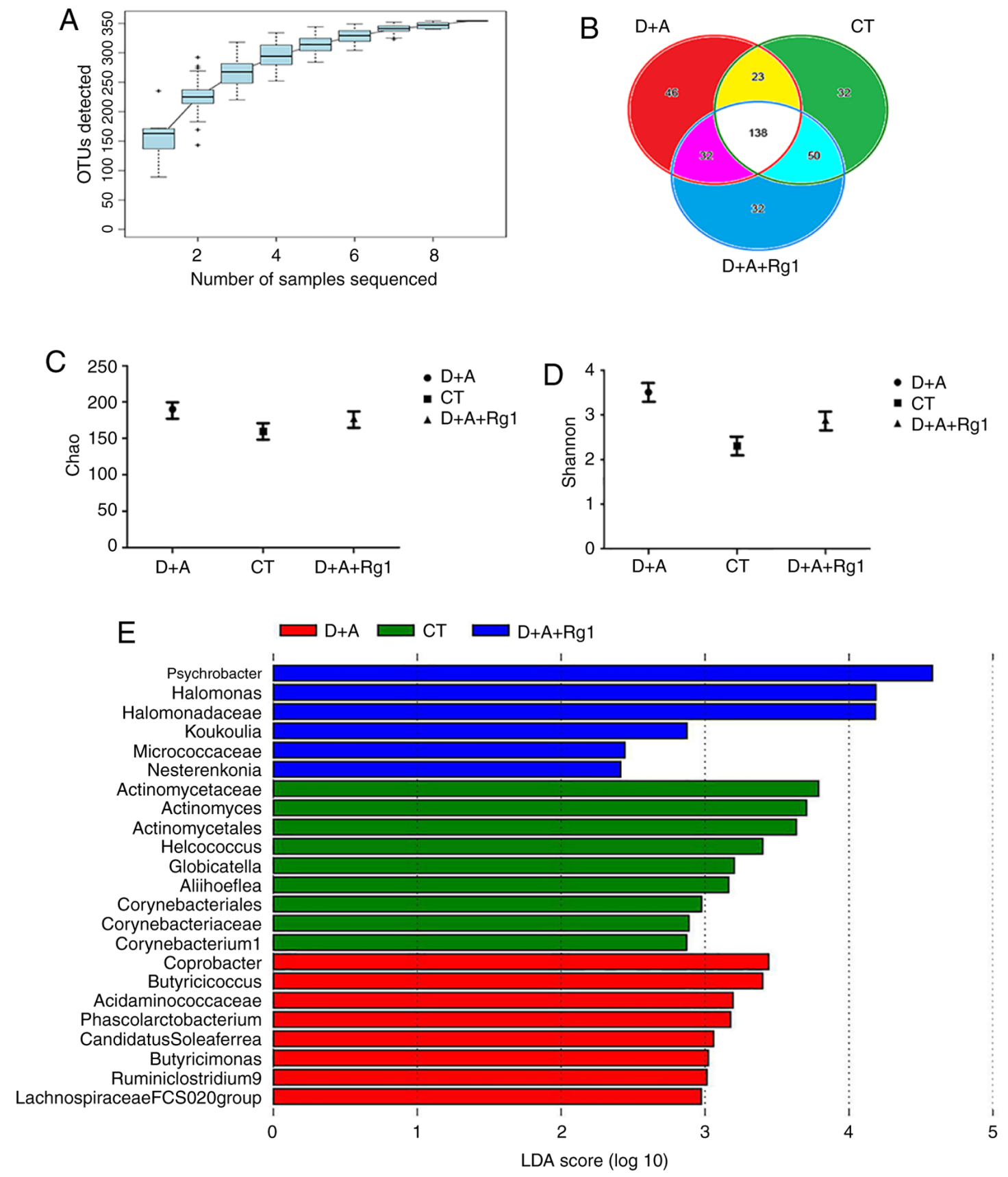

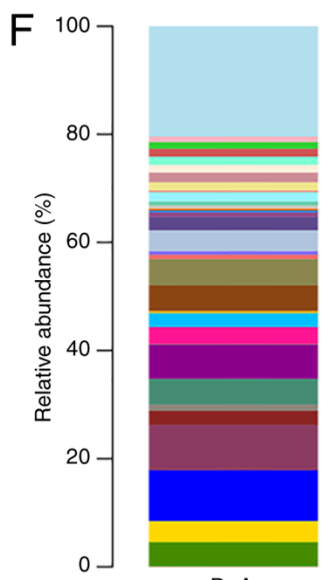

$\mathrm{D}+\mathrm{A}$

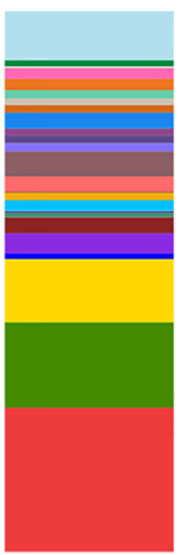

CT

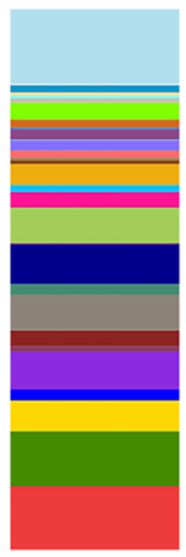

$\mathrm{D}+\mathrm{A}+\mathrm{Rg} \mathrm{l}$
- Lactobacillus salivarius
Lachnoclostridium_uncultured bacterium
a [Ruminococcus] gnavus group_uncultured bacterium
Erysipelatoclostridium_uncultured bacterium
Collinsella_uncultured bacterium
Lactobacillus-uncultured bacterium
Bacteroides_uncultured organism
Streptococcus porcor
Collinsella tanakaei
- Psychrobacter_uncultured bacterium
- Megamonas_Unclassified
Haemophilus influenzae
- Enterobacter_Unclassified
Prevotella 9 uncultured bacterium
Streptococcus uncultured bacterium
Bropides_uncultured bacterium
Bacteroides coprophilus
- Bifidobacterium_Unclassified
- Lactobacillus_Unclassified
Enterococcus faecalis
Enterococcus_Unclassified
ㅁ Halomonas_Unclassified

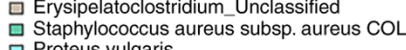
Bacillus thuringiensis
$\checkmark$ Lactococcus_Unclassified
sobacterium uncultured organism
ㅁ Acinetobacter_Unclassified
ㅁ Lachnospiraceae_uncultured bacterium
․ Tyzzerella Unclassified
Morganella morganii subsp. morgani
$\square[$ [Eubacterium] coprostanoligenes group uncultured bacterium $\square$ Megamonas_uncultured bacterium
- Staphylococcus Unclassified
O Others

Alloprevotella_uncultured organism

Figure 5. Analysis of intestinal bacteria. (A) Species accumulation curves. (B) OTUs sharing relationship. Index analysis of (C) Chao and (D) Shannon. (E) LDA score of linear discriminant analysis effect size analysis of tree shrew intestinal flora in the three groups. (F) The analysis of intestinal flora in the genus level. OTUs, operational taxonomic unit; D+A, model group; CT, control group; D+A+Rg1, treatment group; Rg1, ginsenoside Rg1; LDA, linear discriminant analysis. 
is an important component of the neuronal cytoskeleton, serving an important role in different stages of nervous system development, formation and regeneration (36). Jiang et al (37) demonstrated that the decrease of MAP2 in dendrites shows that neurodegeneration leads to neuron death. Neurodegeneration and neuron loss are features of the hippocampus and cortex in $\mathrm{AD}$, making them potential targets for AD treatment strategies (38). Kandimalla et al (39) demonstrated that the accumulation of phosphorylated tau in the hippocampus results in abnormal mitochondrial dynamics and reduces the dendritic protein MAP2, resulting in learning and memory impairment (40).

$\mathrm{NeuN}$ is often used to detect the survival rate of cerebral cortex neurons (36). Leplus et al (41) suggested that increased expression of $\mathrm{NeuN}$ decreases neuronal loss in transgenic AD rats. Intestinal microbiota inducing the low growth phenotype is associated with decreases in the NeuN of humanized gnotobiotic mice (42). The results of the present study demonstrated that Rg1 had protective effects on hippocampal and cortical neurons.

Bcl-2 and Bax are apoptosis-related proteins and the main mechanism of AD causing neuron loss is apoptosis. Bcl-2 is an anti-apoptotic protein, while Bax is a pro-apoptotic protein that enhances programmed cell death (43). Hu et al (44) found that Bax is significantly downregulated and $\mathrm{Bcl}-2$ expression is upregulated following $\mathrm{Rg} 1$ treatment in rats. When damaged, the intestinal villi of a hindlimb unloading rat model upregulates the expression of Bax while downregulating Bcl-2, influencing the expansion of Bacteroidetes and the decrease of Firmicutes in intestinal microbiota (45). Therefore, the expression of Bax and Bcl-2 are associated with Firmicutes and Bacteroidetes.

Rgl alters the microbiota abundance of the large intestine in tree shrews. Previous studies have identified differences in the intestinal microbial composition of neurodegenerative diseases and microbial ecological imbalance may lead to disease pathogenesis (46). The abundance of Bacteroidetes is increased in patients with AD (47). Changes in the microbial composition may alter signals from microorganisms to the central nervous system (48). In the present study, the number of Bacteroidetes in group $\mathrm{D}+\mathrm{A}$ was significantly higher compared with the CT group $(\mathrm{P}<0.01)$, which was consistent with the patients with AD (9). However, Rg1 significantly reduced the abundance of Bacteroidetes in the intestinal tract of tree shrews and there were no significant differences compared with the CT group. These results indicated that $\mathrm{Rg} 1$ can regulate intestinal flora disorder in AD tree shrews.

Vogt et al (47) identified that the abundance of Firmicutes is decreased and Bacteroidetes is increased in patients with AD. The Firmicutes/Bacteroidetes ratio is one of the energy output indicators of microbial fermentation (49); the higher the ratio, the higher the energy output. The proportion of Bacteroidetes decreases and Firmicutes increases in newly malnourished mice at the growth stage after supplementary feeding (50). Furthermore, the Firmicutes/Bacteroidetes ratio increases in the intestinal flora of obese mice (49), increasing the ability to obtain energy from the diet, and the amount of body fat. Zhang et al (8) noted that compared with normal mice, the Firmicutes/Bacteroidetes ratio in the intestinal flora of AD mice of all ages decreased. In the present study, the Firmicutes/Bacteroidetes ratio in the tree shrews of group $\mathrm{D}+\mathrm{A}$ was only 0.0526 times that of the CT group. Following Rg1 treatment, the Firmicutes/Bacteroidetes ratio increased to 6.923 times that of the D+A group. This suggested that the lower Firmicutes/Bacteroidetes ratio of AD tree shrews was related to the reduced energy requirements of $\mathrm{AD}$ tree shrews.

Rezaeiasl et al (51) suggested that Lactobacillus supplementation may positively effect learning capacity in rats with AD. Lactobacillus improves the gut microbiota of a Drosophila melanogaster AD model (52). Lactobacillus salivarius, a gram-positive bacterium, is a type of beneficial bacteria widely existing in the intestinal tract of human beings and animals (53). Feeding with Lactobacillus salivarius can extend the average life span of Caenorhabditis elegans by $11.9 \%$ and increase the activity of superoxide dismutase (53). In the present study, the abundance of Lactobacillus salivarius in the CT group was 24 times higher compared with the D+A group, while that in the treatment group was 10 times higher than the D+A group. It indicates that Rg1 may improve cognitive impairment by increasing the population of Lactobacillus salivarius.

In conclusion, Rg1 can inhibit the expression of pro-apoptotic proteins and protect neurons in a tree shrew AD model. $\mathrm{Rg} 1$ also reduced the production of $\mathrm{A} \beta$ and tau phosphorylation, improved cognitive impairment and the microbiota imbalance of the large intestine. Although the mechanisms of Rg1 in tree shrew AD models remain to be elucidated, the present study provided clues to further elucidate the improvement of cognitive impairment.

\section{Acknowledgements}

Not applicable.

\section{Funding}

This study was supported by the Applied Basic Research Foundation of Yunnan Province [grant nos. 2017FE468-014, 2017FE468-016 and 2019FE001(-026)], the National Natural Science Foundation of China (grant no. 81460647), the Scientific Research Foundation of Yunnan Education Bureau (grant no. 2018Y048) and the Innovation Project of Kunming Medical University (grant no. 2019S093).

\section{Availability of data and materials}

The 16S rRNA database analyzed during the current study are available in the NCBI repository (SRP: 227299; https://www. ncbi.nlm.nih.gov/sra.

\section{Authors' contributions}

YG and LW performed the experiments, and were major contributors in writing the original draft. JL and YY analyzed the data. JJ and HZ helped with data analysis and designed and produced the graphs and edited the manuscript. ZL and $\mathrm{HZ}$ conceived and designed the study. All authors read and approved the final manuscript. 


\section{Ethics approval and consent to participate}

The animal experiment protocol was approved by the Committee on the Ethics of Animal Experiments of Kunming Medical University (approval number KMMU2018004).

\section{Patient consent for publication}

Not applicable.

\section{Competing interests}

The authors declare that they have no competing interests.

\section{References}

1. Quigley EMM: Microbiota-brain-gut axis and neurodegenerative diseases. Curr Neurol Neurosci Rep 17: 94, 2017.

2. Lecouvey G, Morand A, Gonneaud J, Piolino P, Orriols E, Pélerin A, Ferreira Da Silva L, de La Sayette V, Eustache F and Desgranges B: An impairment of prospective memory in mild Alzheimer's disease: A ride in a virtual town. Front Psychol 10: 241-241, 2019.

3. Manso-Calderón R, Cacabelos-Pérez P, Sevillano-García MD Herrero-Prieto ME and González-Sarmiento R: The impact of vascular burden on behavioural and psychological symptoms in older adults with dementia: The BEVASDE study. Neurol Sci 41: 165-174, 2019.

4. Ratno Budiarto B and Chan WH: Oxidative stresses-mediated apoptotic effects of ginsenoside Rb1 on pre- and post-implantation mouse embryos in vitro and in vivo. Environ Toxicol 32: 1990-2003, 2017.

5. Tian W, Chen L, Zhang L, Wang B, Li XB, Fan KR, Ai CH, Xia X, Li SD and Li Y: Effects of ginsenoside Rg1 on glucose metabolism and liver injury in streptozotocin-induced type 2 diabetic rats. Genet Mol Res 16: March 30, 2017. https://doi org/10.4238/gmr16019463.

6. Ong WY, Farooqui T, Koh HL, Farooqui AA and Ling EA: Protective effects of ginseng on neurological disorders. Front Aging Neurosci 7: 129, 2015.

7. Chen LM, Lin N, Zhang J, Zhu YG and Chen XC: Mechanism of ginsenoside $\mathrm{Rg} 1$ regulating the activity of $\beta$ secretase in $\mathrm{N} 2 \mathrm{a} /$ APP695 cells. Zhonghua Yi Xue Za Zhi 92: 330-335, 2012 (In Chinese).

8. Zhang L, Wang Y, Xiayu X, Shi C, Chen W, Song N, Fu X, Zhou R, Xu YF, Huang L, et al: Altered gut microbiota in a mouse model of Alzheimer's disease. J Alzheimers Dis 60: 12411257,2017

9. Claesson MJ, Cusack S, O'Sullivan O, Greene-Diniz R, de Weerd H, Flannery E, Marchesi JR, Falush D, Dinan T, Fitzgerald $\mathrm{G}$, et al: Composition, variability, and temporal stability of the intestinal microbiota of the elderly. Proc Nat Acad Sci USA 108 (Suppl 1): 4586-4591, 2011.

10. Claesson MJ, Jeffery IB, Conde S, Power SE, O'Connor EM, Cusack S, Harris HMB, Coakley M, Lakshminarayanan B, O'Sullivan $\mathrm{O}$, et al: Gut microbiota composition correlates with diet and health in the elderly. Nature 488: 178-184, 2012.

11. Odamaki T, Kato K, Sugahara H, Hashikura N, Takahashi S, Xiao JZ, Abe F and Osawa R: Age-related changes in gut microbiota composition from newborn to centenarian: A crosssectional study. BMC Microbiol 16: 90, 2016.

12. McElhanon BO, McCracken C, Karpen S and Sharp WG: Gastrointestinal symptoms in autism spectrum disorder: A metaanalysis. Pediatrics 133: 872-883, 2014.

13. Pfeiffer RF: Gastrointestinal dysfunction in Parkinson's disease. Lancet Neurol 2: 107-116, 2003.

14. Severance EG, Yolken RH and Eaton WW: Autoimmune diseases, gastrointestinal disorders and the microbiome in schizophrenia: More than a gut feeling. Schizophr Res 176: 23-35, 2016.

15. Doraiswamy PM, Leon J, Cummings JL, Marin D and Neumann PJ: Prevalence and impact of medical comorbidity in Alzheimer's disease. J Gerontol A Biol Sci Med Sci 57: M173-M177, 2002.

16. Kowalski $\mathrm{K}$ and Mulak A: Brain-gut-microbiota axis in Alzheimer's disease. J Neurogastroenterol Motil 25: 48-60, 2019.
17. Mosher KI and Wyss-Coray T: Microglial dysfunction in brain aging and Alzheimer's disease. Biochem Pharmacol 88: 594-604, 2014.

18. Chen SG, Stribinskis V, Rane MJ, Demuth DR, Gozal E, Roberts AM, Jagadapillai R, Liu R, Choe K, Shivakumar B, et al: Exposure to the functional bacterial amyloid protein curli enhances alpha-synuclein aggregation in aged fischer 344 rats and Caenorhabditis elegans. Sci Rep 6: 34477-34477, 2016.

19. Minter MR, Zhang C, Leone V, Ringus DL, Zhang X, OylerCastrillo P, Musch MW, Liao F, Ward JF, Holtzman DM, et al: Antibiotic-induced perturbations in gut microbial diversity influences neuro-inflammation and amyloidosis in a murine model of Alzheimer's disease. Sci Rep 6: 30028-30028, 2016

20. Harach T, Marungruang N, Duthilleul N, Cheatham V, Mc Coy KD, Frisoni G, Neher JJ, Fåk F, Jucker M, Lasser T, et al: Reduction of Abeta amyloid pathology in APPPS1 transgenic mice in the absence of gut microbiota. Sci Rep 7: 41802-41802, 2017.

21. Zhang CH, Sheng JQ, Sarsaiya S, Shu FX, Liu TT, Tu XY, Ma GQ, Xu GL, Zheng HX and Zhou LF: The anti-diabetic activities, gut microbiota composition, the anti-inflammatory effects of Scutellaria-coptis herb couple against insulin resistance-model of diabetes involving the toll-like receptor 4 signaling pathway. J Ethnopharmacol 237: 202-214, 2019.

22. Sun YF, Zhang X, Wang XY and Jia W: Effect of long-term intake of ginseng extracts on gut microbiota in rats. Zhongguo Zhong Yao Za Zhi 43: 3927-3932, 2018 (In Chinese).

23. Cheng D, Chang H, Ma S, Guo J, She G, Zhang F, Li L, Li X and Lu Y: Tiansi liquid modulates gut microbiota composition and tryptophan-kynurenine metabolism in rats with hydrocortisoneinduced depression. Molecules 23: 2832, 2018.

24. Xiao J, Liu R and Chen CS: Tree shrew (Tupaia belangeri) as a novel laboratory disease animal model. Zool Res 38: 127-137, 2017.

25. Fan Y, Luo R, Su LY, Xiang Q, Yu D, Xu L, Chen JQ, Bi R, Wu DD, Zheng P, et al: Does the genetic feature of the Chinese Tree Shrew (Tupaia belangeri chinensis) support its potential as a viable model for Alzheimer's disease research? J Alzheimers Dis 61: 1015-1028, 2018.

26. Zheng H, Niu S, Zhao H, Li S and Jiao J: Donepezil improves the cognitive impairment in a tree shrew model of Alzheimer's disease induced by amyloid- $\beta 1-40$ via activating the BDNF/TrkB signal pathway. Metab Brain Dis 33: 1961-1974, 2018.

27. Römer S, Bender H, Knabe W, Zimmermann E, Rübsamen R, Seeger $\mathbf{J}$ and Fietz SA: Neural progenitors in the developing Neocortex of the Northern Tree Shrew (Tupaia belangeri) show a closer relationship to gyrencephalic primates than to lissencephalic rodents. Front Neuroanat 12: 29, 2018.

28. Health N: Guide for the care and use of laboratory animals. NIH contract No No1-RR-2-2135 1985:11-28.

29. Ghumatkar PJ, Patil SP, Peshattiwar V, Vijaykumar T, Dighe V, Vanage $\mathrm{G}$ and Sathaye S: The modulatory role of phloretin in A 325-35 induced sporadic Alzheimer's disease in rat model. Naunyn Schmiedebergs Arch Pharmacol 392: 327-339, 2019.

30. Chao A: Non-parametric estimation of the classes in a population. Scand J Stat 11: 265-270, 1984

31. Du H, Guo L, Zhang W, Rydzewska M and Yan S: Cyclophilin D deficiency improves mitochondrial function and learning/ memory in aging Alzheimer disease mouse model. Neurobiol Aging 32: 398-406, 2011.

32. Feng L, Liao YT, He JC, Xie CL, Chen SY, Fan HH, Su ZP and Wang Z: Plasma long non-coding RNA BACE1 as a novel biomarker for diagnosis of Alzheimer disease. BMC Neurol 18: 4, 2018.

33. Mulder SD, van der Flier WM, Verheijen JH, Mulder C, Scheltens P, Blankenstein MA, Hack CE and Veerhuis R: BACE1 activity in cerebrospinal fluid and its relation to markers of $A D$ pathology. J Alzheimers Dis 20: 253-260, 2010.

34. Dash R, Emran TB, Uddin MMN, Islam A and Junaid M: Molecular docking of fisetin with AD associated AChE, ABAD and BACE1 proteins. Bioinformation 10: 562-568, 2014.

35. Song XY, Hu JF, Chu SF, Zhang Z, Xu S, Yuan YH, Han N, Liu Y, Niu F, He X, et al: Ginsenoside Rg1 attenuates okadaic acid induced spatial memory impairment by the GSK3 $3 /$ tau signaling pathway and the $A \beta$ formation prevention in rats. Eur $J$ Pharmacol 710: 29-38, 2013.

36. Manczak M, Kandimalla R, Yin X and Reddy PH: Hippocampal mutant APP and amyloid beta-induced cognitive decline, dendritic spine loss, defective autophagy, mitophagy and mitochondrial abnormalities in a mouse model of Alzheimer's disease. Hum Mol Genet 27: 1332-1342, 2018. 
37. Jiang S, Nandy P, Wang W, Ma X, Hsia J, Wang C, Wang Z, Niu M, Siedlak SL, Torres S, et al: Mfn2 ablation causes an oxidative stress response and eventual neuronal death in the hippocampus and cortex. Mol Neurodegener 13: 5, 2018.

38. Lax N, Fainstein N, Nishri Y, Ben-Zvi A and Ben-Hur T: Systemic microbial TLR2 agonists induce neurodegeneration in Alzheimer's disease mice. J Neuroinflammation 17: 55, 2020

39. Kandimalla R, Manczak M, Yin X, Wang R and Reddy PH: Hippocampal phosphorylated tau induced cognitive decline dendritic spine loss and mitochondrial abnormalities in a mouse model of Alzheimer's disease. Hum Mol Genet 27: 30-40, 2018.

40. Koo YS, Kim H, Park JH, Kim MJ, Shin YI, Choi BT, Lee SY and Shin HK: Indoleamine 2,3-dioxygenase-dependent neurotoxic kynurenine metabolism contributes to poststroke depression Induced in mice by ischemic stroke along with spatial restraint stress. Oxid Med Cell Longev 2018: 2413841, 2018.

41. Leplus A, Lauritzen I, Melon C, Kerkerian-Le Goff L, Fontaine D and Checler F: Chronic fornix deep brain stimulation in a transgenic Alzheimer's rat model reduces amyloid burden, inflammation, and neuronal loss. Brain Struct Funct 224 363-372, 2019.

42. Lu J, Lu L, Yu Y, Cluette-Brown J, Martin CR and Claud EC: Effects of intestinal microbiota on brain development in humanized gnotobiotic mice. Sci Rep 8: 5443, 2018.

43. Zhong L, Tong Y, Chuan J, Bai L, Shi J and Zhu Y: Protective effect of ethyl vanillin against $\mathrm{A} \beta$-induced neurotoxicity in PC12 cells via the reduction of oxidative stress and apoptosis. Exp Ther Med 17: 2666-2674, 2019.

44. Hu J, Gu Y and Fan W: Rg1 protects rat bone marrow stem cells against hydrogen peroxide-induced cell apoptosis through the PI3K/Akt pathway. Mol Med Rep 14: 406-412, 2016.

45. Jin M, Zhang H, Zhao K, Xu C, Shao D, Huang Q, Shi J and Yang $\mathrm{H}$ : Responses of intestinal iucosal barrier functions of rats to simulated eeightlessness. Front Physiol 9: 729, 2018.

46. Sharon G, Sampson TR, Geschwind DH and Mazmanian SK: The central nervous system and the gut microbiome. Cell 167: 915-932, 2016.
47. Vogt NM, Kerby RL, Dill-McFarland KA, Harding SJ, Merluzzi AP, Johnson SC, Carlsson CM, Asthana S, Zetterberg H, Blennow K, et al: Gut microbiome alterations in Alzheimer's disease. Sci Rep 7: 13537-13537, 2017.

48. Heiss CN and Olofsson LE: The role of the gut microbiota in development, function and disorders of the central nervous system and the enteric nervous system. J Neuroendocrinol 31: e12684, 2019.

49. Turnbaugh PJ, Ley RE, Mahowald MA, Magrini V, Mardis ER and Gordon JI: An obesity-associated gut microbiome with increased capacity for energy harvest. Nature 444: 1027-1031, 2006.

50. Preidis GA, Ajami NJ, Wong MC, Bessard BC, Conner ME and Petrosino JF: Microbial-derived metabolites reflect an altered intestinal microbiota during catch-up growth in undernourished neonatal mice. J Nutr 146: 940-948, 2016.

51. Rezaeiasl Z, Salami M and Sepehri G: The effects of probiotic Lactobacillus and Bifidobacterium strains on memory and learning behavior, long-term potentiation (LTP), and some biochemical parameters in $\beta$-amyloid-induced rat's model of Alzheimer's disease. Prev Nutr Food Sci 24: 265-273, 2019.

52. Tan FHP, Liu G, Lau SA, Jaafar MH, Park YH, Azzam G, Li Y and Liong MT: Lactobacillus probiotics improved the gut microbiota profile of a Drosophila melanogaster Alzheimer's disease model and alleviated neurodegeneration in the eye. Benef Microbes 11: 79-89, 2020.

53. Zhao Y, Zhao L, Zheng X, Fu T, Guo H and Ren F: Lactobacillus salivarius strain FDB89 induced longevity in Caenorhabditis elegans by dietary restriction. J Microbiol 51: 183-188, 2013. 